\title{
Fano filters based on transferred silicon nanomembranes on plastic substrates
}

\author{
Zexuan Qiang, ${ }_{1}^{1}$ Hongjun Yang, ${ }^{1}$ Li Chen, ${ }^{1}$ Huiqing Pang, ${ }^{2}$ Zhenqiang Ma, ${ }^{2, a)}$ and \\ Weidong Zhou ${ }^{1, b)}$ \\ ${ }^{1}$ Department of Electrical Engineering, NanoFAB Center, University of Texas at Arlington, Texas 76019, \\ USA \\ ${ }^{2}$ Department of Electrical and Computer Engineering, University of Wisconsin-Madison, Wisconsin 53706, \\ USA
}

(Received 30 May 2008; accepted 26 July 2008; published online 13 August 2008)

\begin{abstract}
We report here the characteristics of surface-normal optical filters based on Fano resonances on patterned single crystalline silicon nanomembranes (SiNMs), which were fabricated and transferred onto transparent plastic substrates using a SiNM wet transfer process. Detailed experimental and theoretical analyses were carried out on the angular- and polarization-dependent transmission properties. The filter transmission is independent of the incident beam polarization under surface-normal conditions. Angle-independent transmission was observed for specific Fano resonances with certain polarizations. The measured angle-dependent transmission agrees well with the simulated transmission and dispersion properties based on the propagation wave-vector analysis. (C) 2008 American Institute of Physics. [DOI: 10.1063/1.2971199]
\end{abstract}

Photonic crystal slabs (PCSs), with in-plane periodic modulation of dielectric constant introduced in a high-index guiding layer, offer one of the most promising platforms for large-scale on-chip photonic integration. The out-of-theplane optical mode coupling is feasible with the Fano or guided resonance effect, ${ }^{1,2}$ where these in-plane guided resonances above the lightline are also strongly coupled to the out-of-the-plane radiation modes due to phase matching provided by the periodic lattice structure. ${ }^{3}$ In recent years, devices based on Fano resonance such as narrowband filters ${ }^{1-3}$ or broadband reflectors ${ }^{4}$ have attracted great attention.

Crystalline semiconductor nanomembranes (NMs) (Refs. 5 and 6) offer unprecedented opportunities for unique electronic and photonic devices for vertically stacked high density photonic/electronic integration. The SiNM on flexible substrate also enables high performance photonic components being developed for high-density photonic/electronic integration and for flexible photonics. We report here the experimental demonstration of ultracompact Fano filters based on transferred patterned SiNMs on flexible polyethylene terephthalate (PET) substrates.

Photonic crystal (PC) patterned structures were first fabricated on silicon-on-insulator (SOI) wafers. The schematic and scanning electron micrographs (SEMs) of the patterned PC SOI structures are shown in Figs. 1(a) and 1(b), respectively. The patterned PC SOI structure was subsequently transferred onto a PET substrate based on a wet transfer process, ${ }^{7}$ as shown schematically in Fig. 1(c). The quality of the transferred SiNM PC sample was verified with optical diffraction pattern measurements, ${ }^{7}$ with well defined diffraction patterns shown in Fig. 1(d), with the bottom inset showing a micrograph of the transferred SiNM PC sample on the PET substrate under test.

The simulated dispersion plot is shown in Fig. 2(a) for the square lattice PC patterned SiNM on the PET substrate.

\footnotetext{
a) Electronic mail: mazq@engr.wisc.edu.

b)Electronic mail: wzhou@uta.edu.
}

The refractive indices of silicon and PET substrate used in the simulation are 3.464 and 1.5 , respectively. The ratio between the air hole radius $(r)$ and the PC lattice constant $(a)$ is 0.19 . The thickness of the SiNM $(t)$ is $260 \mathrm{~nm}$. Three Fano resonance modes are identified as $\omega_{1}, \omega_{2}$, and $\omega_{3}$, respectively.

The simulated and measured Fano filter transmission characteristics at surface-normal conditions are shown in Fig. 2(b). The experiment was conducted with an unpolarized focused broadband quartz tungsten halogen (QTH) lamp source. The focused beam size is $\sim 150 \mu \mathrm{m}$ in diameter. The transmission of SiNM is normalized to that of the PET substrate (as reference). The target wavelength is $1.56 \mu \mathrm{m}$ (denoted as $\lambda_{1}$ ). There are two other less profound dip/peaks that can be observed around $1.42 \mu \mathrm{m}\left(\lambda_{2}\right)$ and $1.41 \mu \mathrm{m}$ $\left(\lambda_{3}\right)$, respectively. All these three Fano resonance modes agree very well with the simulated transmission results using three-dimensional (3D) finite-difference time-domain spec-
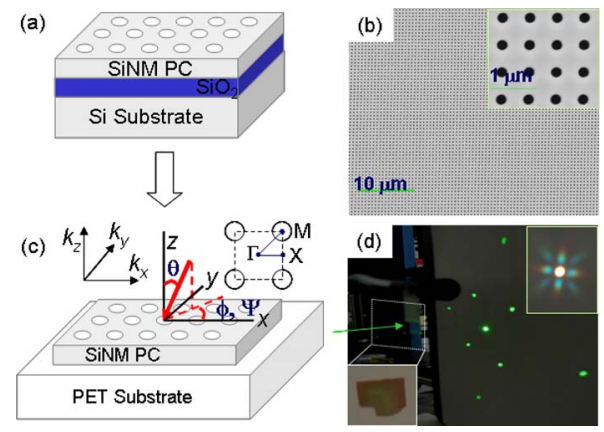

FIG. 1. (Color online) (a) Schematic and (b) SEM of PC patterned structure on the SOI wafer. The zoom-in of the square latter patterns is shown in the inset of (b). (c) Schematic of patterned SiNMs transferred on PET substrate, with the beam incident angles defined. The PC lattice and Brillion zone symmetric points $(\Gamma, X$, and $M)$ in $k$-space are also shown in the inset. (d) Measured diffraction patterns through the SiNM on PET sample with either a cw green laser source or a broadband QTH lamp source (top inset). The micrograph of the transferred SiNM on PET is shown in the bottom inset (center golden colored piece). 


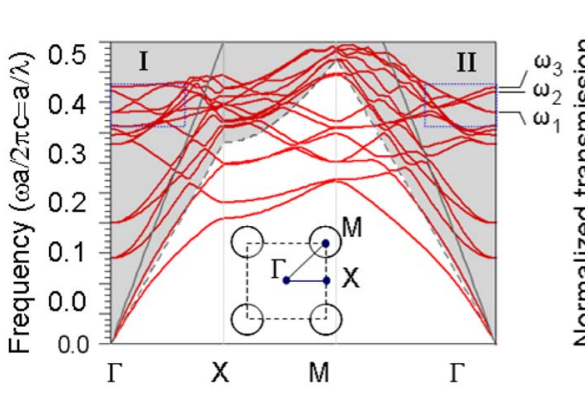

(a)

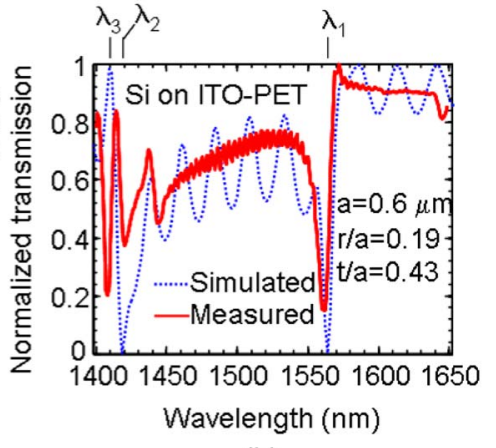

(b)
FIG. 2. (Color online) (a) Simulated dispersion characteristics for the square lattice PC SiNM structure on PET substrate. (b) Measured and simulated surfacenormal transmission $\left(\theta=0^{\circ}\right)$ characteristics for the fabricated patterned SiNM $(t=260 \mathrm{~nm})$ on an ITO-coated PET substrate. Note the surface-normal transmission dips/peaks at three wavelength points $\left(\lambda_{1}, \lambda_{2}, \lambda_{3}\right)$ agree well with the dispersion plot $\left(\omega_{1}, \omega_{2}, \omega_{3}\right)$ at $\Gamma$ point. troscopy [Fig. 2(b)] and also with the simulated dispersion results using $3 \mathrm{D}$ plane wave expansion (PWE) $\left[\omega_{1}, \omega_{2}\right.$, and $\omega_{3}$ in Fig. 2(a) at $\Gamma$ point]. Note that the oscillations in both experimental and simulated transmission spectra shown in Fig. 2(b) are due to the substrate Fabry-Pérot resonances associated with the finite PET substrate thickness $(0.18 \mathrm{~mm})$.

The angular- and polarization-dependent characteristics of Fano filter were also investigated. As shown in Fig. 1(c), the light incident angle are specified by two polar angles, the colatitude angle $\theta$ (angle from the surface-normal direction) and the azimuth angle $\phi$ (angle from the positive $x$ axis to the orthogonal projection of the incident beam in the $x-y$ plane). ${ }^{8}$ The incident beam polarization ( $E$-vector) $\psi$ is defined as the angle from the positive $x$ axis to the polarization direction.

Measured surface-normal $\left(\theta=0^{0}\right)$ transmission results with different angles $\phi$ are shown in Fig. 3, without or with polarizer inserted in between the incident broadband light source and the objective lens in front of the SiNM sample. The $\phi$ angle was changed by rotating the testing sample in the $x-y$ plane as defined in Fig. 1(c). The measured transmission spectra at different angles $\phi$ are shifted along $y$ axis in Fig. 3 for better comparison. Very little spectral shifts were observed on the transmission spectra for all three Fano resonant modes. This is in agreement with previously reported results based on freestanding structures. ${ }^{8}$ The angle $(\phi)$ and polarization $(\psi)$ independent transmission characteristics at $\theta=0^{0}$ are the results of the high symmetry of the circular-airhole square lattice. The small variations on the measured transmission efficiencies could be related to the measurement error. Other factors such as the imperfection of the lattice during the SiNM transfer processes could also lead to small variations in the measured transmission characteristics.

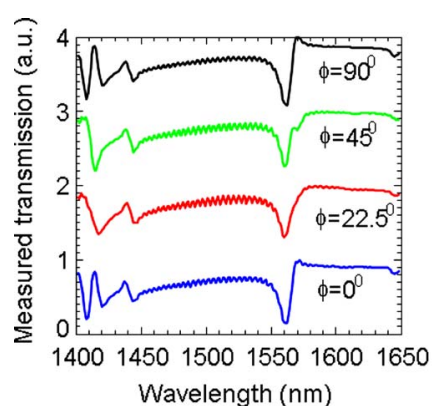

(a)

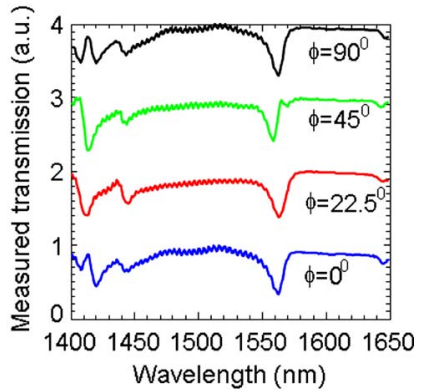

(b)
FIG. 3. (Color online) Measured surface-normal transmission with different angles $\phi$ for (a) without polarizer and (b) with polarizer fixed at $\psi=0$.
However, as the incident angle $\theta$ changes, the Fano resonant modes show different transmission characteristics. A set of transmission curves at different $\theta$ values, when the incident beam was kept in the $x-z$ plane with $\theta=0^{\circ}$ and $\psi=0^{\circ}$ are shown in Fig. 4(a). The dominant Fano resonant mode $\left(\lambda_{1}\right)$ had very little spectral shift over the range of angles that we measured (from 0 to $20^{\circ}$ ), while the other two modes $\left(\lambda_{2}\right.$ and $\lambda_{3}$ ), shifted toward longer wavelengths, with the increase of incident angle $\theta$. As comparison, when the incident beam is kept within the diagonal direction with $\phi=45^{\circ}$ and $\psi$ $=45^{\circ}$ [Fig. 4(b)], both the dominant Fano resonant mode $\left(\lambda_{1}\right)$ and the other two modes $\left(\lambda_{2}\right.$ and $\left.\lambda_{3}\right)$, shifted significantly. All these three modes merge at incident angle of $\theta=20^{\circ}$.

In order to correlate the Fano resonant mode dispersion properties (Fig. 2) with the angle-dependent transmission characteristics (Fig. 4), a detailed analysis was carried out based on the relations between the incident angles and the $k$ vector values in the dispersion plot.

Following the conventional denotations, the irreducible Brillouin zone of the real space square lattice is an isosceles, triangle $(\Gamma X M)$ whose hypotenuse $\Gamma M$ is parallel to the diagonal of each elementary square [Fig. 1(c)]. Considering an incident beam with incident angles denoted as $\theta$ and $\phi$, the corresponding propagation wave vector $k$ in momentum space can be related to the projected components $k_{x}, k_{y}$, and $k_{z}$, as $k_{x}=k \sin \theta \cos \phi, k_{y}=k \sin \theta \sin \phi, k_{z}=k \cos \theta$, and $k$ $=2 \pi / \lambda$, where $\lambda$ is the wavelength in the vacuum.

For the incident beam with wave-vector $k$ kept within the $\Gamma-X$ direction in the $k$-space, i.e., for the case shown in Fig. 4(a) with $\phi=0^{0}$, the momentum vector $k_{x}$ can relate to the normalized momentum $k_{\Gamma-X}$ along $\Gamma-X$ direction as $k_{x}$ $=k_{\Gamma-X}(2 \pi / a) \cos \phi$. Thus the normalized frequency $(a / \lambda)$ can be related to the normalized momentum $k_{\Gamma-X}$ in the dispersion plot as $k_{\Gamma-X}=(a / \lambda) \sin \theta$.

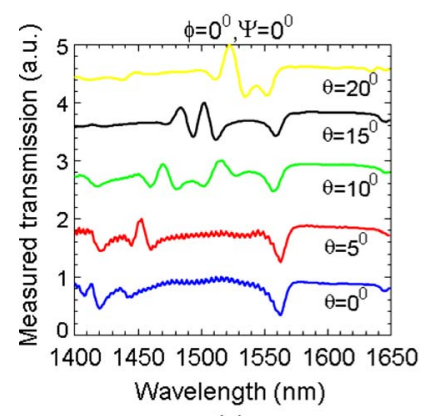

(a)

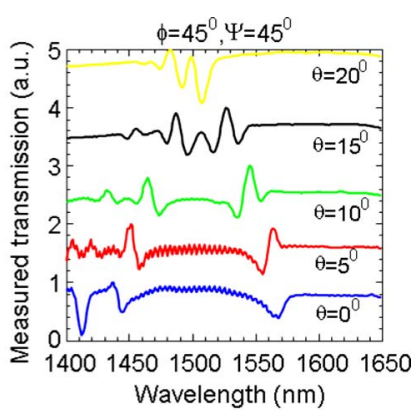

(b)
FIG. 4. (Color online) Measured angle-dependent transmissions with different incident angles $\theta$ for (a) $\phi=0^{\circ}$ and $\psi=0^{\circ}$, and (b) $\phi=45^{\circ}$ and $\psi=45^{\circ}$. 


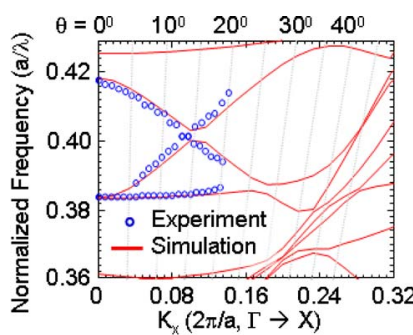

(a)

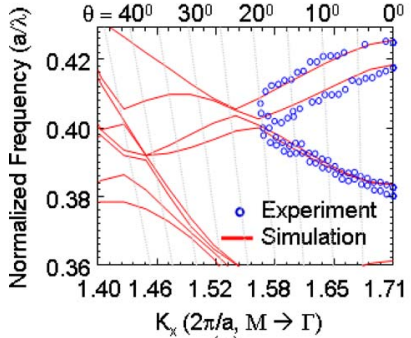

(c)

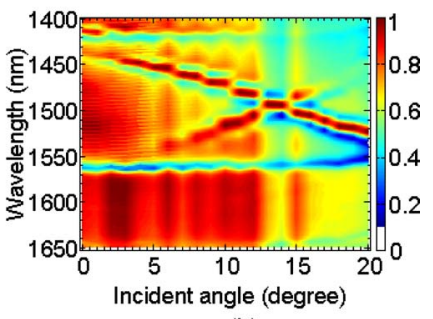

(b)

(d)

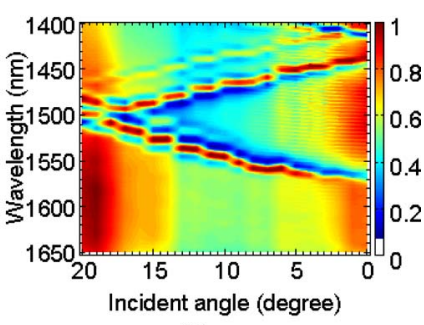

FIG. 5. (Color online) [(a) and (c)] Simulated dispersion plot (red lines) zoomed-in for the regions I and II shown in Fig. 2(a) along $(\Gamma-X, \Gamma-M)$ directions, along with measured Fano resonances (blue dots) for different incident angle $\theta$ with (a) $\phi=0^{\circ}$ and $\psi=0^{\circ}$, and (c) $\phi=45^{\circ}$ and $\psi=45^{\circ}$. The straight gray lines represent the relationships between the normalized frequency and the normalized wave vectors along $(\Gamma-X, \Gamma-M)$ directions. [(b) and (c)] Measured transmission intensity contour plots for angle-dependent transmissions at different incident angle $\theta$ with (b) $\phi=0^{\circ}$ and $\psi=0^{\circ}$, and (d) $\phi=45^{\circ}$ and $\psi=45^{\circ}$

A set of straight lines can be superimposed on the normalized dispersion plot, as shown in Fig. 5(a), which is the zoom-in plot of region I shown in Fig. 2(a), along the $\Gamma-X$ direction. The measured Fano resonant modes were normalized and also plotted in Fig. 5(a) (blue circles). Excellent agreement was achieved between the experiment and simulation results. The transmission intensity contour plots for the measured angle-dependent transmission spectra with $\phi=0^{\circ}$ (Fig. 4) are also shown in Fig. 5(b), where the intensity dips/ peaks are shown as the deep blue and red colors. The inten- sity contour plot clearly reassembles the similar spectra shifting behavior shown in Fig. 5(a).

Following the similar procedure, the measured transmission results shown in Fig. 4(b), with $\phi=45^{\circ}$, were analyzed with wave vector $k$ lies in the $\Gamma-M$ plane, with results shown in Figs. 5(c) and 5(d), respectively. Again, the measurement and the simulation results agree very well.

In conclusion, we fabricated Fano filters based on transferred SiNM on PET substrates. Detailed experimental and theoretical analyses were carried out on the angular- and polarization-dependent transmission properties. The filter transmission is independent of the incident beam polarization under surface-normal conditions. Angle-independent transmission was observed for specific Fano resonances with certain polarizations. The measured angle-dependent transmission agrees well with the simulated transmission and dispersion properties based on the propagation wave-vector analysis.

The authors appreciate the support from NSF (DMI0625728 and DMR-0520527), AFOSR (FA9550-06-1-0482 and FA9550-06-1-0487), AFRL/CONTACT (FA8650-07-25061), and TSGC Programs. W.Z. appreciates the helpful discussions with Dr. Shanhui Fan, Dr. Richard Soref, and Dr. Robert Magnusson. The authors also thank the fabrication support from the University of Texas at Arlington NanoFAB Center and the University of Texas Austin MRC center, part of NSF NNIN sites.

${ }^{1}$ S. Fan and J. D. Joannopoulos, Phys. Rev. B 65, 235112 (2002).

${ }^{2}$ R. Magnusson and S. S. Wang, Appl. Phys. Lett. 61, 1022 (1992).

${ }^{3}$ Y. Kanamori, T. Kitani, and K. Hane, Appl. Phys. Lett. 90, 031911 (2007).

${ }^{4}$ W. Suh and S. Fan, Appl. Phys. Lett. 84, 4905 (2004).

${ }^{5}$ S. A. Scott and M. G. Lagally, J. Phys. D 40, R75 (2007).

${ }^{6}$ H. C. Yuan, Z. Ma, M. M. Roberts, D. E. Savage, and M. G. Lagally, J. Appl. Phys. 100, 013708 (2006).

${ }^{7}$ H. Yang, H. Pan, Z. Qiang, Z. Ma, and W. Zhou, Electron. Lett. 44, 858 (2008).

${ }^{8}$ V. Lousse, W. Suh, O. Kilic, S. Kim, O. Solgaard, and S. Fan, Opt. Express 12, 1575 (2004). 